\title{
A semi-automated method for measuring thickness and white matter integrity of the corpus callosum
}

\author{
S Andronikou, B S Spottiswoode, N Tomazos
}

Department of Radiology, Faculty of Health Sciences, University of the Witwatersrand, Johannesburg $\mathrm{S}$ Andronikou, MB BCh, FCRad, FRCR, PhD

MRC/UCT Medical Imaging Research Unit, Department of Human Biology, University of Cape Town; and Department of Radiology, Stellenbosch University

B S Spottiswoode, $\mathrm{PhD}$

Faculty of Commerce, Department of Management Studies, University of Cape Town

$\mathrm{N}$ Tomazos, BA

Corresponding author: S Andronikou (docsav@mweb.co.za)

\begin{abstract}
Aim. Diseases affecting cerebral white matter may lead to left-right asymmetries and atrophy of interhemispheric connections, i.e. the corpus callosum (CC). Our aim was to describe and test a semi-automated system that divides the midline CC into a number of segments and determines thickness at each, then performs fibre tracking from these segments.

Methods. Six normal female volunteers (average age $25.8 \pm 6.7$ years) and a female patient with diagnosed multiple sclerosis (age 26 years) were scanned on a 3T MRI. We performed diffusion-weighted imaging in 12 directions, and calculated diffusion tensors and fractional anisotropy (FA) maps from this pre-processed data. Fibre tracking from a region-of-interest encompassing the entire CC was done. This fibre data, together with FA maps and the unweighted diffusion tensor imaging (DTI) image $\left(b=0 \mathrm{~s} / \mathrm{mm}^{2}\right)$, were imported into a custom tool written in MATLAB. The midline sagittal position was carefully defined by selecting multiple midline points in coronal and axial views and rotating the image volume and fibre co-ordinates accordingly.

Using the customised tool, dorsal and ventral CC contours were manually drawn on the mid-sagittal FA image, initiating automated calculation of a contour midway between these manually drawn lines. The programme was designed to then divide the midline contour into a pre-selected number of segments; from each segment border, perpendicular spokes were projected until they intersected with the dorsal and ventral contours. This technique divided the CC into a pre-set amount of segments, the number of which was limited by the spatial resolution. It was decided to set the number at 40 to ensure that each segment depicted a contiguous strip of voxels across the $\mathrm{CC}$ from the dorsal to the ventral contour. The system allows these segments to then be used as seeds for separate fibre tracking in each cerebral hemisphere, and various parameters are automatically plotted as a function of distance along the midline contour. The following parameters are measurable: midline CC thickness; midline FA; fibre volume for each hemisphere (represented as a left/right ratio centred on zero) and mean fibre FA for each hemisphere (also represented as a left/ right ratio centred on zero).

Results. The tool proved successful in measuring and plotting CC midline thickness and FA, but was not sensitive for peripheral white matter lesions.

Conclusions. The technique successfully determined values of CC midline thickness, FA and interhemispheric differences. Future research will determine normal values for age and compare CC thickness with peripheral white matter volume loss in large groups of patients, using the semiautomated technique.
\end{abstract}

S Afr J Rad 2012;16(4):130-133. DOI:10.7196.SAJR.652

Many diseases affect human brain white matter, multifocally or diffusely, in adults and children. These may be metabolic (e.g. Leigh's disease), toxic (e.g. carbon monoxide poisoning), hypoxic (e.g. hypoxic ischaemic injury), infective (e.g. HIV or syphilis), inflammatory (e.g. multiple sclerosis) or vascular (e.g. microvascular dementia). Two important conditions that result in significant loss of global white matter volume in affected children are hypoxic ischaemic injury and HIV encephalopathy. These diseases result in the thinning of associated fibre bundles and may be reflected by atrophy of inter-hemispheric connections - most importantly the corpus callosum (CC) (Fig. 1).

A simple automated method of determining normal values of the CC according to age is desirable. A tool which is also applicable for diagnosing white matter disorders and offering prognosis in children with global white matter insults will have a wide clinical application. This article describes a semi-automated system that divides the midline CC into a number of segments, determines thickness at each, and performs fibre tracking from these segments. 

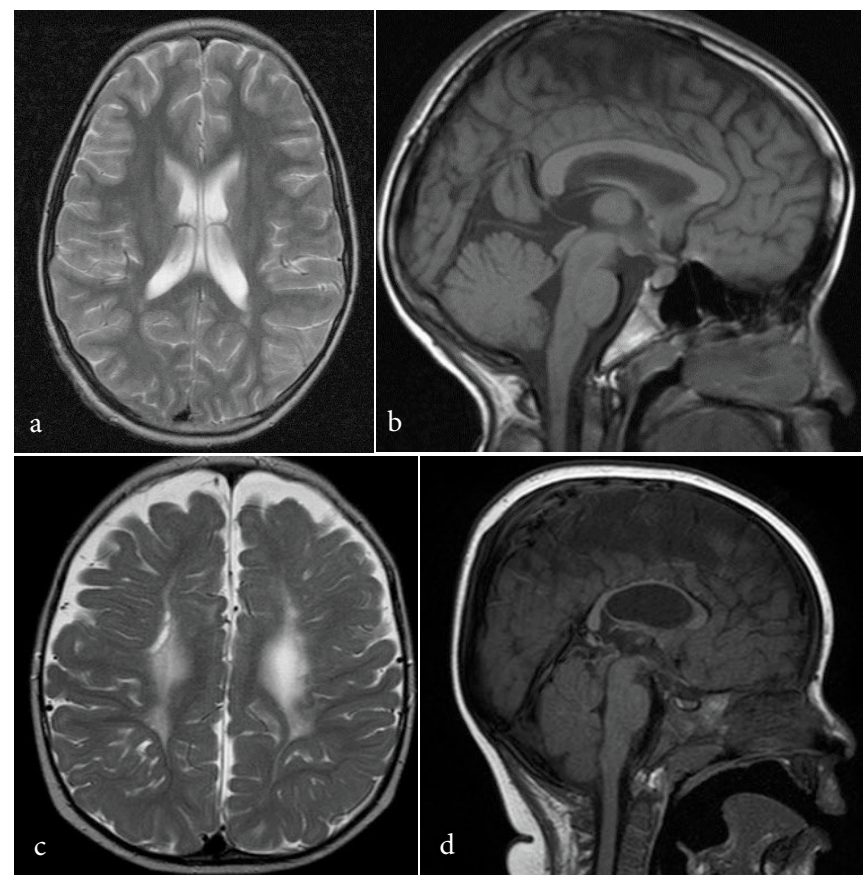

Fig. 1( $a$ and $b)$. A child with a normal volume of peri-ventricular white matter on (a) axial T2-weighted imaging and (b) the corresponding corpus callosum thickness expected on the sagittal image. Fig. 1(c and d) demonstrates a child with a previous global hypoxic insult affecting the brain and resulting in marked white matter volume loss. This is best appreciated on the axial T2-weighted image (c) by the depth of the sulci, which abut the ventricular edge. The corresponding sagittal image (d) shows easily identifiable thinning of the corpus callosum in response to this extreme white matter volume loss.

\section{Methodology}

Six normal female volunteers (average age $25.8 \pm 6.7$ years) and a 26-year-old female patient with diagnosed multiple sclerosis (MS) were scanned on a 3T MRI system (MAGNETOM Allegra, Siemens, Erlangen). Informed consent was obtained and protocols were approved by the Stellenbosch University Ethics Committee.

Diffusion-weighted imaging was performed in 12 directions with a b-value of $1000 \mathrm{sec} / \mathrm{mm}^{2}$, and one volume was acquired with $\mathrm{b}=0 \mathrm{sec} /$ $\mathrm{mm}^{2}$. Other parameters included:

- $\quad$ field of view $(\mathrm{FOV})=240 \mathrm{~mm}$

- $\quad$ spatial resolution $=1.9 \times 1.9 \mathrm{~mm}^{2}$

- $\quad$ slice thickness $=1.9 \mathrm{~mm}$

- $\quad$ matrix size $=128 \times 128$ pixels

- $\quad$ slices $=75$

- $\mathrm{TR}=10400 \mathrm{~ms}$

- $\mathrm{TE}=86 \mathrm{~ms}$

Five identical acquisitions were taken, and were averaged after eddy correction and outlier rejection. Co-registration was then performed across the 5 averages using affine transformations, with the unweighted image of the first average as a reference. Diffusion tensors and fractional anisotropy (FA) maps were then calculated from these pre-processed data.

From a region-of-interest encompassing the entire CC, fibre tracking was done using fibre assignment by continuous tracking (FACT) in DTIStudio (Jiang and Mori, Johns Hopkins University, 2007). The FA tract initiation and termination threshold was 0.2 , and the principle eigenvector angle stopping criterion was $70^{\circ}$. Extraneous fibres, such as those projecting down into the spinal cord, were removed. This fibre data, together with FA and the unweighted diffusion tensor imaging (DTI) image $\left(b=0 \mathrm{~s} / \mathrm{mm}^{2}\right)$, were imported into a custom tool written in MATLAB (The Mathworks Inc, Natick, MA). The midline sagittal position was carefully defined by selecting multiple midline points in coronal and axial views, and rotating the image volume and fibre co-ordinates accordingly.

Using the customised tool, dorsal and ventral CC contours were manually drawn on the mid-sagittal FA image. This initiated the automated calculation of a contour midway between the manually drawn lines. The programme was designed to then divide the midline contour into a pre-selected number of segments, from the borders of which perpendicular spokes were projected until they intersected with the dorsal and ventral contours. This in turn divided the CC into a preset number of segments (Fig. 2). The number of segments was limited by the spatial resolution, and it was decided that having 40 segments would ensure that each one still contained a contiguous strip of voxels across the CC from the dorsal to the ventral contour.

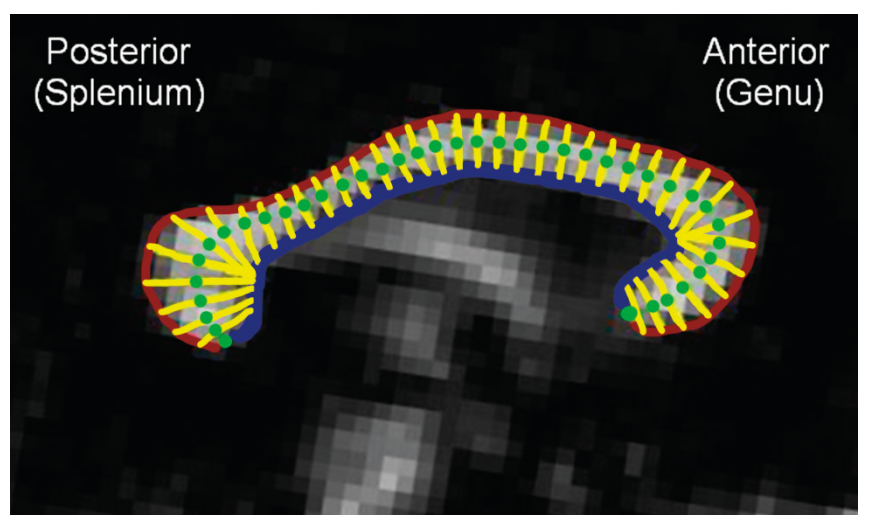

Fig. 2. Semi-automated division of the corpus callosum into multiple intervals along a midline contour. The corpus callosum superior (red) and inferior (blue) contours were drawn manually by the user on the sagittal DTI image. The midline contour (green) was calculated automatically using the software, and lines perpendicular to the midline were extended (again automatically) until they intersected with the manual contours. These perpendiculars offer the measures of corpus callosum thickness at several locations and also serve as seeds for fibre tracking.

With this system, the segments can be measured (representing the CC thickness at several locations) and used as seeds for fibre tracking separately in each cerebral hemisphere (Fig. 3). The various parameters are automatically plotted as a function of distance along the midline contour. The following parameters are measurable:

- midline CC thickness

- midline FA

- fibre volume for each hemisphere (represented as a left/right ratio centred on zero)

- mean fibre FA for each hemisphere (also represented as a left/ right ratio centred on zero).

\section{Results}

Fig. 4 shows the various parameters successfully determined from the MS patient, plotted as a function of normalised distance along the CC midline, with the splenium to the left and the genu to the right. The 


\section{ORIGINAL ARTICLE}

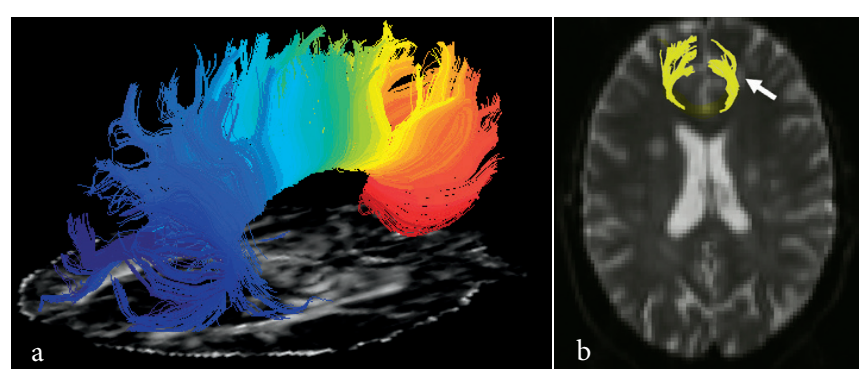

Fig. 3. Fibre tracking can be performed for all the regions simultaneously and is colour-coded by distance along the midline contour (a) or for isolated regions as selected by the user (b).

dotted lines provide a normal range (defined as the mean \pm 1 standard deviation of the corresponding parameters) successfully determined from the 6 normal subjects. To simplify interpretation but still preserve the trend details, these normal bounds have been smoothed using a 6 th order polynomial.

\section{Discussion}

The CC is the main inter-hemispheric commissure of the brain. It plays a role in unifying sensory fields, organising bimanual motor output, aiding memory storage and retrieval, allocating attention and arousal, facilitating language and auditory functions, and in consciousness itself. ${ }^{1}$ For pathologists and radiologists alike, however, the ability to evaluate the volume and integrity of white matter has been notoriously elusive. Now, DTI fibre tracking, and DTI-derived metrics such as FA, provide a means for measuring and demonstrating white matter features. ${ }^{2}$ DTI fibre tracking can be used to associate known anatomical landmarks (such as the CC) with regions where localisation may be ambiguous (such as off-midline, or peripheral, hemispheric white matter). ${ }^{3}$

The midline sagittal view of the $\mathrm{CC}$ has been parcellated in a variety of ways according to both structural geometry and function..$^{4-8}$ DTI fibre tracking has already been used in this context to segment the CC into bands corresponding to structural and functional units. ${ }^{9-11} \mathrm{CC}$ thickness in the sagittal plane has been shown to be associated with a variety of factors including better motor performance ${ }^{12}$ and with verbal IQ and fluency. ${ }^{13}$ The CC thickness may be secondarily affected by fibre loss, ${ }^{12}$ as seen in children after severe hypoxic ischaemic injury, HIV encephalopathy or large infarcts.

The semi-automated system that we designed (as described above) was tested successfully and allowed measurement of midline CC, midline FA, mean fibre FA for each hemisphere and fibre volume for each hemisphere for all 7 adults. These were plotted by the system as graphs demonstrating the ability to determine ranges of normal values. This would be suitable for use in children, to determine agerelated differences in myelin maturation and volume. The technique can now also be used to compare CC thickness with hemispheric brain volume in both normal children and those with cerebral atrophy, such as seen with hypoxic ischaemic injury and HIV encephalopathy. Observations can then be correlated with functional scores and used for predicting outcome and function. In addition, cases in which patients with HIV are receiving antiretroviral therapy can be followed up to determine if the patients regain white matter volume and CC thickness.

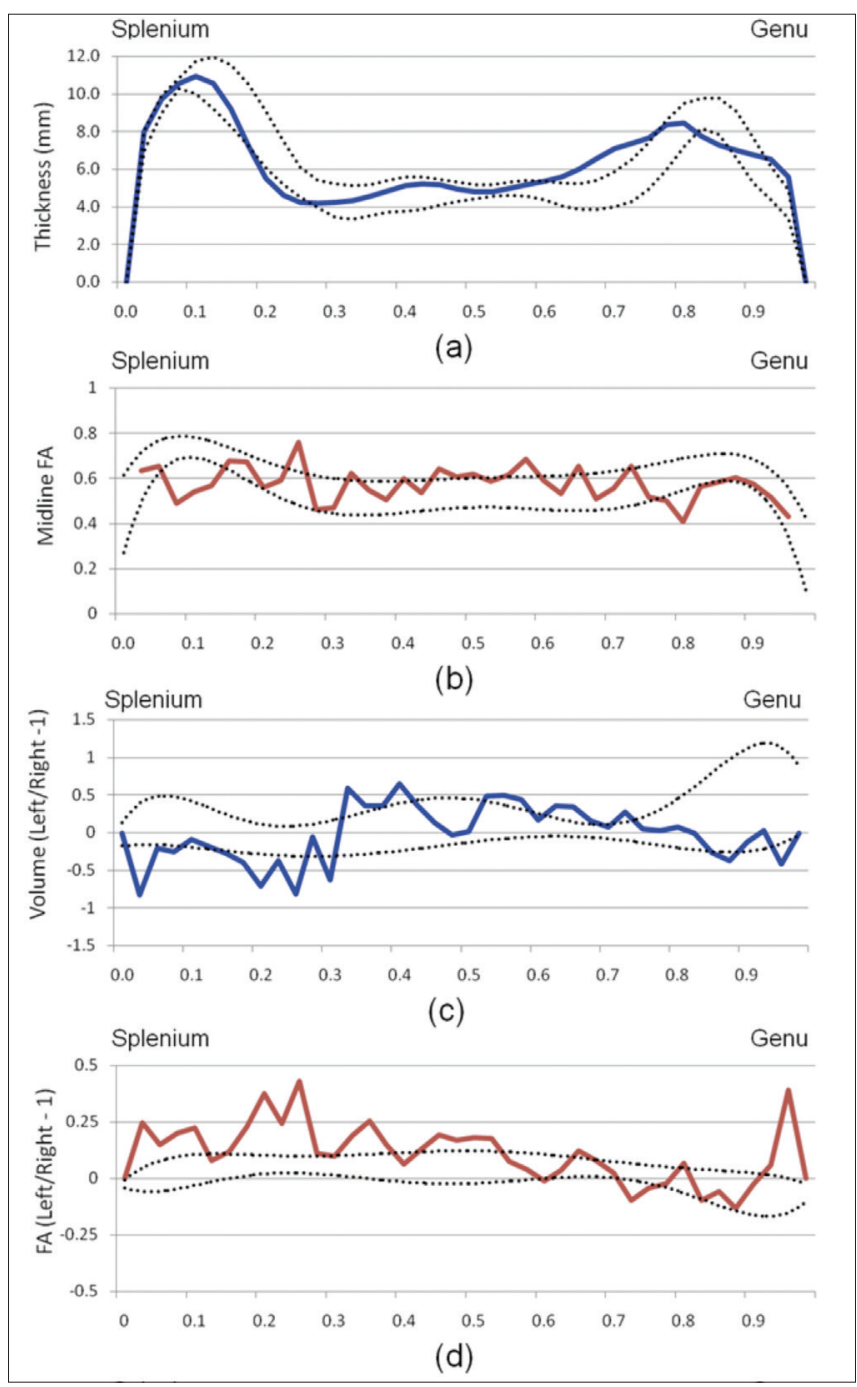

Fig. 4. The result of the semi-automated system is a battery of automatically generated graphs, plotting patient parameters as a function of the normalised distance along the corpus callosum midline (demonstrated here for the patient with multiple sclerosis). (a) Corpus callosum thickness; (b) fibre volume (left/ right-1); (c) mean fibre FA (left/right-1); and (d) midline FA. The dotted lines in (a) indicate corresponding mean \pm 1 standard deviation values for the 6 normal subjects' corpus callosum thickness.

For the patient with MS, we anticipated a thinning of the CC or decrease in midline FA. A previous study by Dorion et al. ${ }^{14}$ shows the total size of the CC in females to be unrelated to hemispheric asymmetry. In our patient, differences in CC thickness (Fig. 4a) were too large to be accounted for by differences in midline contour length. Although regional decreases in FA in normal-appearing white matter have been shown in the CC of MS patients ${ }^{15}$, there is no clear indication thereof in Fig. 4b.

On a macro-scale, a healthy brain is largely symmetrical across the mid-sagittal plane. ${ }^{16}$ However, the ratio of grey to white matter is smaller in the right hemisphere than in the left, ${ }^{17}$ and numerous regional interhemispheric asymmetries have also been identified. ${ }^{17-21}$ Of these inter-hemispheric differences, regions that the CC fibres are likely to cross include frontal areas (where the right volume is greater than the left) and 
occipital areas (where the left volume is greater than the right). Even with rough estimations of the location of the frontal and occipital regions, these differences are not apparent in the normal ratios in Fig. 4c.

Fig. $4 \mathrm{~d}$ shows a more consistent hemispheric bias, suggesting that the left hemispheric CC fibres have a consistently greater FA than the corresponding fibres in the right hemisphere. This is compatible with the theory that the left hemisphere is focally organised, whereas the right is more diffusely organised. ${ }^{22} \mathrm{~A}$ voxel-based asymmetry analysis, using FA images, also identified several regions in the CC where FA was greater in the left hemisphere than in the right. ${ }^{23}$ The FA provides a convenient measure of macro-axonal organisation and its integrity, and there is histological evidence suggesting that regional axonal loss in the CC correlates with peripheral white matter lesion volume and distribution. ${ }^{24}$

\section{Conclusions}

Our study aims to provide a semi-automated means of evaluating peripheral white matter volume and integrity using inter-hemispheric tract symmetry and features measured at the midline CC as a surrogate marker. The technique has been tested on an MS patient and 6 normal, healthy volunteers. The tool has proven that it can be used to determine normal values including the thickness and FA of the CC, as well as differential hemispheric white matter fibre volume at several locations. The tool shows good promise for measuring the CC thickness and plotting abnormal patients against a calculated normal range. Future work planned will compare CC thickness, measured using this technique, with brain volume (segmented into white matter and grey matter) in an attempt to use CC thickness as an indicator of white matter volume loss in children with HIV and hypoxic ischaemic injury.

Acknowledgements. We thank Siemens Medical Solutions South Africa and all the staff at the Cape Universities Brain Imaging Centre.

1. Giedd JN, Rumsey JM, Castellanos FX, et al. A quantitative MRI study of the corpus callosum in children and adolescents. De Brain Res 1996;91(2):274-280.

2. Beaulieu C. The basis of anisotropic water diffusion in the nervous system - a technical review. NMR Biomed 2002;15:435-455.
3. Berman JI, Mukherjee P, Partridge SC, et al. Quantitative diffusion tensor MRI fibre tractography of sensorimotor white matter development in premature infants. Neuroimage 2005;27(4):862-871.

4. Witelson SF. Hand and sex differences in the isthmus and genu of the human corpus callosum. Brain 1989;112:799-835.

5. Hynd GW, Semrud-Clikeman M, Lorys AR, et al. Corpus callosum morphology in attention deficithyperactivity disorder: morphometric analysis of MRI. J Learn Disab 1991;24:141-146.

6. Rajapakse JC, Gieff JN, Rumsey JM, et al. Regional MRI measurements of the corpus callosum: a methodological and developmental study. Brain Dev 1996;18:379-388. [http://dx.doi.org/10.1016/03877604(96)00034-4]

7. Denenberg VH, Kertesz A, Cowell PE. A factor analysis of the human's corpus callosum. Brain Res 1991;548(1-2):126-132.

8. Peters M, Oeltze S, Seminowicz D, et al. Division of the corpus callosum into subregions. Brain Cogn 2002;50:62-72.

9. Dougherty RF, Ben-Shachar M, Deutsch G, et al. Occipital-callosal pathways in children: validation and atlas development. Ann NY Acad Sci 2005; 1064:98-112.

10. Hofer S, Frahm J. Topography of the human corpus callosum revisited - comprehensive fibre tractography using diffusion tensor magnetic resonance imaging. Neuroimage 2006;32(3):989-994.

11. Hasan KM, Kamali A, Iftikhar A, et al. Diffusion tensor tractography quantification of the human corpus callosum fibre pathways across the lifespan. Brain Res 2009;1249:91-100.

12. Skranes J, Vangberg TR, Kulseng S, et al. Clinical findings and white matter abnormalities seen on diffusion tensor imaging in adolescents with very low birthweight. Brain 2007;130:654-666. [http://dx.doi. org/10.1093/brain/awm001]

13. Yung A, Poon G, Qiu DQ, et al. White matter volume and anisotropy in preterm children: a pilot study of neurocognitive correlates. Pediatr Res

14. Dorion AA, Chantôme M, Hasboun D, et al. Hemispheric asymmetry and corpus callosum morphometry: a magnetic resonance imaging study. Neurosci Res 2000;36(1):9-13.

15. Hasan KM, Gupta RK, Santos RM, Wolinsky JS, Narayana PA. Diffusion tensor fractional anisotropy of the normal-appearing seven segments of the corpus callosum in healthy adults and relapsing remitting multiple sclerosis patients. J Magn Reson Imaging 2005;21(6):735-743.

16. Joshi S, Lorenzen P, Gerig G, Bullitt E. Structural and radiometric asymmetry in brain images. Med Image Anal 2003;7:155-170. [http://dx.doi.org/10.1016/S1361-8415(03)00002-1]

17. Petty RG. Structural asymmetries of the human brain and their disturbance in schizophrenia. Schizophr Bull 1999;25:121-139.

18. Galaburda AM, LeMay M, Kemper TL, Geschwind N. Right-left asymmetries in the brain. Science 1978;199:852-856.

19. Shapiro R, Galloway SJ, Shapiro MD. Minimal asymmetry of the brain: a normal variant. Am J Roentgenol 1986;147(4):753-756

20. Iwasaki S, Nakagawa H, Fukusumi A, et al. Left-right asymmetry of the temporal and parietal regions in children: based on the medullary pattern of cerebral white matter. Surg Radiol Anat 1990;12:209-214.

21. Watkins KE, Paus T, Lerch JP, et al. Structural asymmetries in the human brain: a voxel-based statistics analysis of 142 MRI scans. Cereb Cortex 2001;11(9):868-877.

22. Semmes J. Hemispheric specialisation: A possible clue to mechanism. Neuropsychologia 1968;6:11-26. [http://dx.doi.org/10.1016/0028-3932(68)90035-3]

23. Park H, Westin C, Kubicki M, et al. White matter hemisphere asymmetries in healthy subjects and in schizophrenia: a diffusion tensor MRI study. Neuroimage 2004;23(1):213-223.

24. Evangelou N, Konz D, Esiri MM, et al. Regional axonal loss in the corpus callosum correlates with cerebral white matter lesion volume and distribution in multiple sclerosis. Brain 2000;123:18451849. 